\title{
Los Satélites de Transmisión Directa en México: el caso de las antenas parabólicas
}

\author{
Javier Esteinou Madrid \\ Departamento de Educación y Comunicación, Universidad Autónoma \\ Metropolitana Xochimilco
}

\section{Panorama}

Con la inauguración del Sistema Morelos de Satélite: (SMS) en 1985, México ingresó al reducido circulo de países que contaban con infraestructura satelital de uso doméstico, para efectuar sus comunicaciones internas. Dicho sistema permitió al país, por primera vez en la historia, mantenerse comunicado desde todos los puntos de territorio nacional. Por eso 1985 es un año crucial en e desarrollo de las telecomunicaciones nacionales, pues marcó una nueva etapa en el desarrollo de la infraestructura de la: comunicaciones de la República Mexicana.

Aunado a este nuevo fenómeno, surgió en el país en la década de los ochenta otro de similares magnitudes que, por lo mismo, es necesario tenerlo en consideración. Nos referimos a la transmisión directa de señales vía satélite, es decir aquella comunicación que se establece desde una estación emisora ubicada en el espacio con un sin número de receptores individuales distribuidos en distintas zonas geográficas de la tierra.

La transmisión directa implica una forma de comunicación distinta a las utilizadas por México en décadas anteriores. Para realizarla ya no son necesarias las extensas redes de microondas, ni las complicadas estaciones retransmisoras. Ahora, sólo se requiere la colocación exterior de Una antena receptora en forma de parábola, cuyo diámetro depende de la región en donde vaya a ser instalada y de la función específica que vaya a desempeñar.

Debido al éxito y a la rápida expansión de dichas actividades (Antenas parabólicas) en el país, la industria y el comercio de materiales y equipos de recepción directa, hoy en día, son dos sectores sumamente dinámicos que crecen con gran celeridad por toda la República. Incluso, por su alta rentabilidad pueden considerarse como una de las nuevas zonas económicas donde el gran capital busca reproducirse de manera diversificada. 
Si bien es cierto que no se prevé que este nuevo medio llegue a convertirse en masivo a corto plazo (dados los altos costos que representa la compra e instalación de los equipos de recepción), si puede tener una gran influencia en la evolución mental de la vida nacional, e incluso agravar ciertos problemas de carácter cultural, como son la penetración de patrones ideológicos exógenos. Importa, por consiguiente, apuntar que las señales retransmitidas desde el espacio al territorio nacional es, en su totalidad, de procedencia extranjera, principalmente estadounidense.

En este marco, resulta necesario destacar el papel que el Sistema Morelos de Satélites y otros sistemas complementarios deberán jugar con objeto de contrarrestar las transmisiones foráneas y servir de instrumento fortalecedor de la identidad, la cultura y la soberanía nacionales. Por ello, es importante dedicarle mayor atención y estudio a dicho fenómeno.

Con esto, la sociedad mexicana no estará desprevenida ante las consecuencias que se provoquen y el Estado tendrá posibilidades de actuar en defensa de los intereses nacionales, ya que dicha renovación tecnológica será de grandes alcances, no sólo para México, sino para todo el contexto internacional. Por todo lo anterior, es muy importante revisar los aspectos más destacados que han presentado la evolución y impacto de la transmisión directa en los principales ámbitos de la realidad mexicana.

\section{Antecedentes internacionales de la difusión directa}

El acelerado desarrollo tecnológico de la comunicación vía satélite ha traído consigo una nueva forma de tele recepción que ha producido cambios en la comunicación espacial, particularmente, en el caso de los servicios domésticos de radiodifusión directa. (Diagnóstico, s/f: 1680-1681.)

Dicho avance tecnológico implica un cambio profundo en la forma de transmitir y recibir las señales de satélites. Hasta hace algunos años la comunicación espacial funcionaba por medio de estaciones terrenas de grandes dimensiones (aproximadamente 10 metros de diámetro) capaces de captar las señales retransmitidas desde el espacio. Una vez logrado esto, la señal era enviada desde tierra a todo el resto del territorio nacional por medio de redes de microondas enlazadas con estaciones transmisoras y retransmisoras de microondas, de televisión, o con centrales telefónicas ubica- das en poblaciones a las que estaban destinadas. (Diagnóstico, s/f: 1680-1681.)

Posteriormente, el acelerado avance tecnológico permitió la creación de antenas de menores dimensiones y más baratas, que funcionaban adecuadamente como receptores. De este progreso surgió el uso de satélites para servi- 
cios domésticos, ya que al abatir los costos de las repetidoras terrenas, se facilitaba su mayor uso, mediante la colocación de estaciones en diversos puntos estratégicos. Con el aprovechamiento de las características de cobertura de los satélites de haz amplio, los territorios se podían enlazar a las telecomunicaciones en toda su extensión. Cuestión que permitía obtener un ahorro considerable respecto a los sistemas convencionales como el de microondas y, además, en forma global y con mayor eficiencia, los servicios de telecomunicaciones se podrían llevar hasta las regiones más apartadas. (Diagnóstico, sir: 1674)

A la estación terrena base le fueron asignados los enlaces vía microondas y las comunicaciones internacionales. En cambio a las más pequeñas, se les encargó recibir las señales provenientes de los satélites y retransmitirlas en la zona donde estaban ubicadas. Con ambas características se permitió el uso de las comunicaciones espaciales en servicios continuos de telecomunicaciones regionales, especialmente de televisión.

El factor que permitió reducir las dimensiones de los equipos de recepción fue el incremento de la potencia de transmisión de los satélites. Es decir, el hecho de que contaran con mayor capacidad de transmisión, por lo cual, era necesaria una antena de menores dimensiones.

Sin embargo, al introducir las estaciones repetidoras con capacidad para transmitir las señales sin recurrir a las microondas, se puede hablar de un nuevo elemento de distinción: los sistemas satelitales de transmisión directa. Esto es, del satélite a la antena receptora y de ésta al aparato de televisión. Surge así la difusión directa de tipo comunitario, que implica la recepción de señales por medio de equipos receptores destinados al uso de una comunidad, por medio de un sistema de distribución que abarca un área limitada. (Diagnóstico, sir: 1674)

En 1977 se realizó en Japón un experimento que modificó el panorama de las telecomunicaciones, y que es el primer antecedente de la difusión directa por satélite para uso individual. Dicho experimento demostró que mediante el uso de una pequeña antena de un metro de diámetro se podría captar las señales de televisión enviadas desde el espacio por un satélite de transmisión directa. Dicho satélite poseía la potencia adecuada para permitir el uso de una antena de pequeño diámetro para recibir informaciones.

A partir de entonces, Japón efectuó experimentos continuos con el satélites ETS (Kiku), construido por Ford y operado por la Agencia Gubernamental del Japón para cuestiones Espaciales (NASDA), en la que se operaba con antenas de 1.6 metros de diámetro. 
Lo anterior significó, a nivel internacional la irrupción de la comunicación directa individual. Es decir, que mediante una antena instalada en el techo de una vivienda, la señal de un satélite podía ser captada, eliminando la intermediación de equipos comunales de recepción o distribución de señales. Esto requirió también la necesaria participación de las empresas que laboraran en la construcción y lanzamiento de satélites, así como de equipos de telecomunicación; ya que la aparición de esta posibilidad, implica, por un lado, la gradual desaparición del equipo de recepción y distribución comunal y, por el otro, la posibilidad de comercializar, a gran escala, el uso de satélites para televisión doméstica. Tendencia que si bien no se produciría de inmediato, si se vislumbra a largo plazo. En este sentido, las principales empresas estadounidenses de la electrónica por medio de Comsat, así como la Corporación Canadiense de Televisión (CBS) se apresuraron a incorporar dicha innovación a su producción. (Diagnóstico, s/f: 1675)

Tiempo después la Comsat tramitó ante la Comisión Federal de Comunicaciones de Estados Unidos (CDC) la autorización para crear un sistema de transmisión directa individual, y ser, con ello, la primera empresa del mundo que lo hacía. Por otro lado, se presentó un conflicto con Comsat y la Asociación Nacional de Difusoras (AND), ya que la estructuración de la televisión estadounidense se basa en el uso de estaciones locales y de sistemas por cable, y el satélite de difusión directa a los hogares transformaba el concepto de localismo, debido a que la señal enviada por el satélite alcanzaría a toda la nación. Con esto, argumentaban los representantes de la AND, que dejarían de satisfacerse las necesidades del público local. Además, se oponían igualmente a que dicho servicio se autorizara a la Comsat, ya que ello significaría cederle el principal espacio en el espectro electromagnético, para su comercialización.. (Diagnóstico s/f: 1677).

En Octubre de 1979 la CFC derogó el requerimiento que obligaba a poseer licencia para instalar estaciones receptoras de satélites. Con ello, los costos de producción se redujeron significativamente y se aceleró su obtención (la norma que subsistió fue la que establece contar con la aprobación de las entidades operadoras de satélites para poder recibir sus transmisiones). Además, se argumentaba que la aprobación no constituiría más que el paso previo para su instalación, ya que su funcionamiento operativo se iniciaría hasta 1985. Sin embargo, los preparativos para lograr la recepción con antenas pequeñas no cesaban. En 1981 se podían adquirir antenas de menos de 1 metro de diámetro, que costaban entre 400 y 500 dólares, aunque por en fechas no se pudieran utilizar ya que los satélites con la potencia idónea no eran aún lanzados. (Diagnóstico, s/f: 1678) 
Por consiguiente, la tendencia en los últimos años ha sido reducir los costos y dimensiones de las antenas, al tiempo de que se lanzan satélites de mayor potencia de transmisión. Ambas cuestiones, facilitarán no sólo la comercialización de los servicios de recepción, sino también la proliferación de satélites para usos domésticos. Además de los EVA, con objeto de competir internacionalmente, otras naciones se trazaron planes y proyectos para incorporar la recepción directa individual a los servicios domésticos. Entre los principales países destacaron Francia y Alemania Federal con los satélites TOF - 1 y TV -SAT -1, lanzados por el cohete Ariane en 1984. Luxemburgo, por conducto de la empresa privada de radiodifusión más grande de Europa, Compañía Luxemburguesa de Teledifusión (CL T), que tuvo su propio satélite en 1985, con un costo de 185 millones de dólares y cuyo lugar de lanzamiento por el taxi espacial Columbia, fue apartado con 100,000 dólares. (Diagnóstico, s/f: 1679)

Otros países que también se han incorporado a dicho servicio son Japón y Canadá. El primero al lanzar su primer satélite, en 1983; y el segundo a raíz del lanzamiento del Anik B, cuyo contrato fue otorgado por el Departamento de Comunicaciones de Canadá a la empresa Telesat. (Diagnóstico, s/f: 1680) El panorama a futuro muestra una comercialización creciente del servicio, lo que implica una enorme demanda creciente de satélites y la consecuente inversión que esto requiere. Simplemente, se calcula que en la década de los 80's la demanda fue de 40 satélites para la difusión directa, lo que implicó la inversión de 3 billones de dólares, con la participación de la industria Europea en un 50 por ciento. A esto hay que agregar 400 millones de dólares en sistemas de lanzamiento y 25 millones en instrumentos de transmisión y control de tierra. En este aspecto, hay que subrayar la importante participación que desempeñaron los transbordadores espaciales de EUA, que están diseñados para colocar hasta 17 satélites en un solo viaje. Desde 1982 los planes vislumbraban ya la realización de dos viajes espaciales mensuales a partir de ese mismo año. (Diagnóstico, s/f: 1680)

\section{La transmisión directa en México}

De acuerdo con la definición aceptada por la Unión Internacional de Telecomunicaciones (UIT), se entiende por transmisión directa aquel servicio que permite la captación de señales emitidas desde el espacio por un satélite, mediante el uso de instalaciones domésticas sencillas, como antenas de pequeñas dimensiones conectadas a los televisores de cada hogar. (Kanahuati: 1982: 1) 
La principal distinción que hay entre la transmisión directa y la doméstica por medios tradicionales, como las microondas o el cable coaxial, se relaciona no con la forma de transmisión, sino con la forma de recepción de las señales Esto significa, que en la difusión directa las transmisiones se realizan desde un punto ubicado en el espacio hasta otro punto que es el receptor individual de las señales, sin que éstas tengan que pasar en términos físicos y técnicos, por alguna estación retransmisora. Este hecho significa que las señales no pueden ser objeto de ninguna clase de limitación control, reglamentación o interferencia por parte de los Estados nacionales.

Un sistema de satélites de transmisión directa supone la existencia de uno o más artefactos colocados en la órbita geoestacionaria, que tengan entre sus características el ser de "alta potencia". Esto es, que permitan a los usuarios del sistema, captar señales satisfactorias de radiodifusión en las casas habitación, o en cualquier otro lugar donde existan antenas receptoras de un diámetro no mayor de 1 metro en las de tipo individual, y de 2.5 a 4.5 metros de diámetro en las de tipo comunitario. En ambos casos, la antena deberá ser colocada en un sitio despejado del exterior.

Los satélites de difusión directa han sobrepasado ya la etapa de experimentación y diseño. Hoy están en la fase de perfeccionamiento y comercialización. De esta forma, por ejemplo, en 1986 América Latina amplió su red de difusión directa a partir de los satélites norteamericanos puestos en órbita para la región.

El sistema de difusión directa corresponde a una nueva concepción técnica de radiodifusión, en la que la densidad de potencia radiada permite la radiorecepción con instalaciones no profesionales que podrá montar y adquirir el público en general. Las emisiones que puede abarcar el sistema son de televisión, teleaudición y sistemas de transmisión de datos (Vicepresidencia: 1981: 1)

La mayoría de los satélites de telecomunicación en uso en la actualidad no son de difusión directa. Requieren el apoyo de antenas de grandes proporciones, así como de estaciones retransmisoras para que sus señales sean captadas.

las transmisiones de televisón que irradian sobre diversos territorios nacionales pueden ser percibidas individualmente, mediante la colocación exteriror de antenas parabólicas de gran tamaño, de un diámetro de entre 3.5 y 7 metros. La parábola no es más que una red, una fórmula esférica o parabólica; esto es, un medio por el cual las microondas que emite un satélite se pueden concentrar en un punto determinado. Estas llegan, mezcladas al aparao que las recibe, el cual las integra, amplifica, baja y separa. y uuna vez clasificadas en una gama de frecuencias, entran al televisor. 
La antena parabólica debe de estar orientada hacia uno de los satélites geoestacionados que, de acuerdo con su ubicación orbital, están en posibilidades de "bañar" con señales la zona geográfica en donde la antena se encuentre colocada. La antena parabólica, por tanto, sólo podrá recibir las señales y captar los canales de televisión que transmitan por medio de ese satélite.

Entre tanto, los proyectos de difusión directa han avanzado mucho a raíz de la Conferencia Administrativa Regional para la Planificación del Servicio de Radiodifusión por Satélite en la Región 2, realizada en junio y julio de 1983, en la que México obtuvo vasias posiciones orbitales para difusión directa: la 127 grados y la 136 grados de longitud oeste, con protección de eclipse y plena capacidad de canales; y la de 78 grados longitud oeste, sin protección de eclipse y plena capacidad de canales. El total de los 112 canales disponibles tiene un ancho de banda de 24 metros, suficiente para transmitir una señal de televisión en color con codificación compuesta en frecuencia modulada y dos subportadores de sonido, lo cual debe permitir transmisiones de televisión con sonido estereofónico. Las bandas que se atribuyen para el enlace descendentes mghz, y para el enlace ascendente son de $17.3 \mathrm{y}$ 18.8mghz. (Fadul, 1985: 19-20)

Fuera de los Sistema Morelos de Satélites y del Sistema Solidaridad, hasta el momento no se conocen otros proyectos i concretos de parte del gobierno actual para utilizar satélites de difusión directa. En cambio, en relación a la iniciativa privada y más concretamente a la empresa Televisa se habla de un proyecto de satélite panamericano, que será lanzado durante la década de los noventa, "como parte de las celebraciones del Quinto Centenario del Descubrimiento de América. Se pretende colocarlo en una posición de 57 grados Oeste y su más probable constructor será la compañía Hughes Aircraft, utilizando un modelo HS393 de difusión directa”. (Fernández, 1985)

Si se recuerda que en Diciembre de 1982 se modificó el artículo 28 de la Constitución, para definir la comunicación vía satélite como actividad estratégica a cargo total del Estado y si se tiene presente que el 26 de diciembre de 1984 el Congreso aprobó las modificaciones a la Ley de Vías Generales de Comunicación, la cual indica en su artículo 11, que el establecimiento, la operación y el control de sistemas de satélites quedan reservados, en forma exclusiva, al gobierno federal; puede pensarse que, por ahora, Televisa está impedida por ley, para colocar satélites en posiciones orbitales mexicanas.

Sin embargo, no está limitada para ubicarlos en posiciones orbitales de EVA, por lo que desde hace casi un año la empresa citada solicitó permiso por medio de su red en EVA, para construir, lanzar y operar un sistema de satélite panamericano cuya señal puede llegar no sólo a México sino a toda América 
Latina y a las poblaciones de habla hispana y los EVA, sin necesidad de atender a lo que dicta la letra de la Constitución Política de los Estados Unidos Mexicanos. (Fernández, 1985)

Esta realidad tecnológica modifica totalmente el concepto y la realidad de soberanía nacional esbozada hasta ahora, por lo que es necesario estudiar a fondo este fenómeno político cultural y sus repercusiones sobre nuestro país.

Principales compañías fabricantes o importadoras de estaciones terrenas y de equipos electrónicos para recepción vía satélite

El año de 1980 resulta ser una fecha clave en lo que aquí se denomina difusión directa de señales vía satélite en México. En esta fase surgen en el país las primeras antenas parabólicas con capacidad para captar a los satélites de EUJ: y Canadá.

A partir de esa fecha, tanto la fabricación nacional de antenas como la importación de los equipos electrónicos de recepción se han mantenido en constante expansión al grado de que en la actualidad se pueden identificar a por lo meno 14 empresas dedicadas a este nuevo y lucrativo negocio. Entre las principales compañías figuran las siguientes: Diseños Electromecánicos, S.A. (DEMSA), Telesat, S.A. d C.V., Sekure 2000, S.A., Ramsa, S.A., Comercial Arsa, Rafael Mejía y Asociados, A.P., Programatic Nacional S.A., Satelfin, S.A., Videosat, Orbisat, S.A., Orbicom, S.A Serintelc, S.A. y Resalt, S.A.

Todas ellas presentan características similares en cuanto a los equipos y los servicios que ofrecen, los cuales se podrían sintetizar así:

1. Las antenas parabólicas se ofrecen en tres diferente " dimensiones: 3.75, 5 y 7 metros de diámetro, así como en tres materiales diferentes: aluminio, fibra de vidrio y malla metálica. Sus características varían de acuerdo con los deseos del cliente y el lugar de la República Mexicana en donde se les pretenda instalar.

2. Los equipos electrónicos de recepción desde el espacio consisten en: a) Amplificador de bajo ruido (LNA); b) Convertidor de bajada; c) Selector para 24 canales; d) Control remoto polorotor; e) Control remoto para cambiar canales (opcional); f) Restrictor de canales (opcional) y g) Rastreador de satélites (opcional).

3. De acuerdo con las especificaciones que cada empresa presenta, las antenas están en posibilidad de recibir seña- les de satélites de las series Satcom, Westar, Galaxy, Comstar y Anik, de EUA y Canadá, en algunos casos.

4. Los principales servicios televisivos que pueden ser sintonizados son, entre otros: 
1.- Películas internacionales; 2.- Información financiera; 3.- Espectáculos internacionales; 4.- Programación exclusiva para adultos; 5.- Operas y conciertos; 6.-Documentales científicos; 7.- Ultimas noticias sobre política internacional; 8.- Eventos deportivos; 9.- Programas educativos y de entretenimiento para niños; 10.- Programas de orientación vocacional, social, personal y de autodesarrollo para adolescentes, así como emisiones especiales sobre la enseñanza del manejo de computadoras y sus diversos lenguajes; 11.Programación cultural diferida en 30 idiomas las 24 horas del día; 12.- Servicios informativos actualizados sobre turismo internacional; 13.- Programas especiales para autopromoción de la mujer y 14.- Transmisiones en vivo desde la Cámara de Representantes, audiencias directas desde el Congreso de los EUA y seminarios y conferencias políticas durante las 24 horas.

\section{El marco legal de las telecomunicaciones vía satélite en México}

El surgimiento del Sistema Morelos de Satélites es una realidad que coloca a México entre los pocos países que cuentan con un sistema satelital para uso doméstico. Empero, nuestras leyes no preveían este moderno fenómeno del que la Nación ya forma parte. Es por esto que el Gobierno de la República ha comenzado a adquirir conciencia de la vital importancia que tendrán las telecomunicaciones vía Satélite en el desarrollo del país y ha realizado las primeras modificaciones legales que considera pertinentes, con el fin de actualizar el marco jurídico en esta materia y prever algunas consecuencias que traerá dicho acontecimiento para el futuro cultural de México. Bajo estas consideraciones, resulta pertinente una sintética revisión de la legislación mexicana sobre telecomunicaciones espaciales.

$\mathrm{Al}$ respecto, se puede decir que en octubre de 1980 el Presidente de la República, José López Portillo, dio a conocer la autorización a la Secretaria de Comunicaciones y Transportes (SCT) para proyectar un Sistema Nacional de Satélites (SNS). Dicho sistema consistiría de tres artefactos: Uno que se mantuviera en órbita proporcionando un servicio permanente, otro que acompañara al primero en su recorrido y que sería utilizado sólo en los casos en que fallara el sistema, y uno más que permanecería en tierra, como reserva. (Fernández, 1984:16-17)

Una vez efectuada la autorización del Ejecutivo para su creación en junio de 1981 se procedió a concretizar diversas reuniones entre organismos oficiales y la empresa privada. El objetivo de las mismas fue determinar no solo quien seria el fabricante del artefacto y que características técnicas debería poseer, sino también discutir el financiamiento y las instalaciones terrenas necesarias para asegurar la recepción y el mantenimiento del proyecto. 
El 5 de julio de 1982 la SCT y la empresa Televisa, S.A., firmaron un convenio para la "construcción y puesta en órbita de un satélite mexicano de transmisión directa, que haría factible recibir en los hogares de la República Mexica- na la señal televisiva sin necesidad de retransmisión; esto es, de manera directa”. (Fernández, 1984: 16-17) El convenio fue firmado por parte de la SCT, por el entonces titular de esa dependencia, Licenciado Emilio Mújica Montoya y por parte de Televisa S.A., por su presidente y apoderado general señor Emilio Azcárraga Milmo. (Schmuc1er, 1983: 69-71)

Tres meses después celebrado el convenio citado, e14 de octubre de 1982 se dio a conocer a la opinión pública que la empresa que se encargaría de construir el aparato sería la Hughes Comunications International (HCI). Ese mismo día se acordó en el despacho del titular de la SCT, que la fabricación del satélite sería cofinanciada por Televisa S.A.,. (Fernández, 1984) Sin embargo, en diciembre de 1982, con el advenimiento a la presidencia de la República del licenciado Miguel de la Madrid Hurtado, se produjo un cambio significativo en la legislación nacional en materia de telecomunicaciones espaciales.

El gobierno actual por iniciativa del Ejecutivo Federal envió al Congreso un proyecto de ley que preveía la modificación de diversos artículos constitucionales, entre los que destacaban el número 28 . En éste se asienta de manera categórica no solo la rectoría del Estado en materia de comunicaciones vía satélite, sino también se otorga a este el derecho de exclusividad para usarlo. Sobre el punto se decía: "No constituirá monopolios las funciones que el Estado ejerza de manera exclusiva en las áreas estratégicas a las que se refiere este precepto: acuñación de moneda; correos; telégrafos; radiotelegrafía; y la comunicación vía satélite; emisión de billetes por medio de un solo banco, organismos descentralizados del Gobierno Federal: petróleo y los demás hidrocarburos; petroquímica básica; minerales radioactivos y generación de energía nuclear, electricidad, ferrocarriles; y las actividades que expresamente señalen las leyes que expidan el Congreso de la Unión”. (Constitución: 20) De esta manera, quedaron inválidos todos los convenios que se hubieran celebrado en esta materia durante el gobierno precedente.

En 1984, un año antes del lanzamiento y puesta en órbita del Sistema Morelos de Satélites, se intentó elaborar un complemento jurídico para el uso, mantenimiento y operación del sistema satelital. Es por esto que el Presidente Miguel de la Madrid envió a la Cámara de Senadores una iniciativa para modificar el artículo 11 de la Ley de Vías Generales de Comunicaciones. Aceptado el cambio por el senado de la República, la iniciativa fue enviada al Congreso de la Unión, en donde finalmente fue aprobada. 
La iniciativa subrayó lo siguiente: Artículo II. La prestación de los servicios públicos de los sistemas telegráficos, radiotelegráficos y el de correos queda reservada exclusiva- mente al Gobierno Federal. También quedan reservados en forma exclusiva al Gobierno Federal el establecimiento de los sistemas de satélites, su operación y control y el aprovechamiento de estos se llevarán a cabo conforme a las bases que para tal efecto fije la Secretaría de Comunicaciones y Transportes de acuerdo con esta ley y sus reglamentos". (DOF, 1985: 21)

Asimismo señala que a las concesiones para el servicio de Satélites otorgados por la Secretaría de Comunicaciones y Transportes se les fijarán las bases a que deben sujetarse los prestadores de servicios de vías generales de comunicación. En relación a dichas bases el articulado, puntualiza: "la SCT podrá modificar las tarifas cuando el interés público lo exija, oyendo previamente a los prestadores de servicios afectados y siempre que al hacerlo no se comprometa la costeabilidad misma de la explotación. (Gil, 1984)

Junto con el aprovechamiento del Satélite doméstico se ha dado en el país un hecho más de similares dimensiones, que ya esta siendo considerado por nuestras leyes, aunque todavía muy parcialmente. Este otro hecho es el de la captación directa de señales de vía satélite o mediante antenas de plato.

Las antenas parabólicas, podrán ser consideradas como el instrumento indispensable para lograr que las señales provenientes del espacio, sean captadas en tierra. Su aprobación y autorización parte de la Dirección General de Concesiones y Permisos y se sustenta en lo establecido en las fracciones II y III de la Ley Orgánica de la Administración pública General. Los usuarios deberán cumplir, por tanto, con los siguientes requisitos y limitaciones que señala dicha ley a fin de que el permiso correspondiente les sea otorgado:

a.- Las antenas parabólicas o estaciones receptoras no se podrán utilizar para captar y comercializar señales vía satélite.

b.- Su utilización individual, familiar o comunitaria no tendrá ninguna clase de restricciones; salvo el pago de un impuesto anual de \$ 15,000 M.N. (Quince mil viejos pesos M/N.), por posesión de la antena, y cumplir con los requisitos que la Secretaría de Comunicaciones y Transportes exige para la autorización del permiso.

c.- Para obtener el permiso por parte de la SCT se necesita:

1. Llenar la solicitud requerida por la Secretaría y de- mostrar que la estación y los equipos sean bien habidos y se encuentren perfectamente legalizados por las autoridades correspondientes.

2. En el caso que una estación terrena pretenda ser utilizada con fines comerciales, requiere ser concesionada por la SCT, sujetándose a los requisitos y obligaciones que para ello se demanden. 
3. Para hacer uso público de las señales provenientes de satélites extranjeros, se requiere presentar una autorización previa del país o del particular dueño de la señal. Si se pretende hacer uso de la programación de determinado satélite de comunicaciones, el prestador de servicios debe de exhibir el permiso correspondiente. Es necesaria también, la certificación de la Secretaría de Gobernación (SEGOB) en la cual se autoriza a recibir y difundir programas televisivos de origen extranjero. (Barriga, 1982: 94)

Con estas medidas se fortalecen parcialmente algunos planteamientos de la Constitución Política de los Estados Unidos Mexicanos, y el Estado conserva parcialmente la rectoría en materia de telecomunicaciones espaciales dentro de un marco de libertad y un régimen de economía mixta. No obstante éstas supervisiones gubernamentales, aún hace falta que nuestras leyes prevean muchas de las implicaciones políticas y culturales que puede traer consigo la difusión de señales vía satélite, como son los contenidos ideológicos de las transmisiones o la dependencia tecnológica del exterior. Todos estos asuntos son de vital, importancia para conservar y apoyar la identidad cultural y soberanía política de México.

\section{La presentación ideológica}

El rápido ingreso de México en el ámbito de la difusión directa de señales vía satélite ha provocado diversos cambios no solo en lo referente a la comercialización de las estaciones receptoras y los equipos electrónicos; sino también en el ámbito legal, cuyos cambios han sido argumentados ideológicamente con el supuesto de que las señales transmitidas en el espacio no pertenecen a nadie en particular. Bajo este supuesto, lo que se ha buscado es evitar la comercialización del uso o la recepción de esas señales. Para esto, se plantea que "quien tenga el permiso necesario y el dinero suficiente para pagar los derechos del caso, puede instalar libremente una antena de ese tipo, pero no podrá cobrar ni concesionar a terceros este servicio”. (Alvarez, 1984: 4)

Bajo esta racionalidad, ha sido fácil lograr la expansión ,1 y proliferación por todo el país de las empresas dedicadas a fabricar, importar, vender e instalar equipos de recepción de señales provenientes del espacio. Por ello, es muy conveniente llamar la atención al Gobierno Federal y, en especial, a la Secretaría de Gobernación, sobre las consecuencias inesperadas de dicha innovación tendrá en el desarrollo socioeconómico y político del país. 


\section{Las repercusiones sociales de la transmisión directa}

La transmisión directa de señales vía satélite ya es una realidad ampliamente extendida en México. Aunque si bien es cierto que se trata de un fenómeno relativamente reciente en el país, no se puede pensar que, a corto plazo, se convierta en un hecho masivo para toda la nación, dado los altos costos de los equipos de recepción de señales. No obstante ello, con los actuales datos disponibles es importante analizar y prever algunas de las posibles repercusiones que dicha modernización tecnológica puede provocar sobre el panorama cultural de la República.

\section{A).- La identidad cultural y la soberania nacional}

Desde el punto de vista cultural, la proliferación de transmisiones directas a nivel internacional plantea un problema fundamental: el rebase de las fronteras nacionales por parte de las señales vía satélite. Esto significa, que al utilizar determinado país un satélite doméstico, su señal llega también a territorios de otros países. Las características geográficas limítrofes son el factor que determina la existencia de este problema y sólo aquellos países con grandes extensiones nacionales que utilicen sistemas domésticos orientados a su zona central, pero no a sus fronteras, podrán evitar dicho rebase. Actualmente este es el caso de sólo una minoría de países. (Diagnóstico, s/f: 1680-1681)

Además la tendencia en su utilización, en la generalidad de los casos, presenta el empleo de coberturas totales, donde la señal trasciende las fronteras. Por ello, limitar el uso de satélites únicamente a las zonas centrales de los países con amplios territorios, significaría, por una parte, la subutilización tecnológica de esta costosa infraestructura; y por otra, reducir su aplicación en una minoría de regiones del mundo, lo que mermaría, en su mayor parte, las posibilidades de comercialización del sistema. (Diagnóstico, s/f: 1681)

Dicho rebase constituye un problema para los gobiernos de los países que se aprestan a utilizar la nueva forma de radiodifusión, ya que mediante la simple compra de una antena casera un gran número de personas podrían captar la ii señal de varios canales de televisión de otras naciones. R (Diagnóstico, s/f: 1682)

Esto, propicia, de hecho, el fomento del consumo de artículos extranjeros vía publicidad foránea y el que los países de mayor capacidad de producción televisiva transfieran sus programación a los de menor desarrollo en el área audiovisual. Otros conflictos ideológicos aparecerán en los países re- 
ceptores, cuando los centros emisores, apoyados en la lógica de los colosales teleauditorios, difundan su programación en idiomas extranjeros y fomenten la adopción de hábitos y conductas foráneos.

Ello provocará serias interrogante s sobre la conservación de la identidad nacional, elemento indispensable para todo conglomerado humano que pretenda mantenerse integrado como Nación autónoma e independiente. (Diagnóstico, s/f: 1682-1683) Esto significa para México la presencia de una forma más sutil, pero no menos efectiva de intervención extranjera, como es la penetración cultural, la cual es considerada como uno de los métodos más modernos de conquista territorial, junto con el económico y el militar. El imperialismo cultural puede ser considerado como la forma de intervención más peligrosa, por que llega a ejercer un control efectivo en la mente de los hombres, ya que por medio de la información transmitida se conoce la diversidad idiomática y la cultura que cada país tiene, sustituyéndola por modelos ajenos a la forma de actuar y de pensar del pueblo de México. Conviene añadir que dicha información, además de responder a intereses transnacionales, coinciden también con los grandes intereses que sustentan las oligarquías nacionales. (Rodríguez, 1984)

Ante este problema, el único factor que podría limitar el bombardeo con señales de video más allá de las fronteras nacionales sería la codificación de las emisiones por satélite desde el país dueño. Este hecho ha comenzado a preverse en algunos países del mundo, principalmente en los EUA. (Fadul, 1985: 109) "Al respecto se conoce que los productores de programas para los diversos canales satelitales de los EUA han exigido a la Federal Communication Commision, la protección de los derechos de autor mediante el permiso para la codificación de tales señales. De lograrse esto, sería un acontecimiento de enorme importancia para nuestro país, ya que la mayoría de las señales que transmiten los satélites extranjeros provienen de artefactos estadounidenses. De esta manera, las antenas que hoy proliferan en no pocos hogares mexicanos de amplio poder adquisitivo, y en condominios y hoteles acomodados del país, no estarían aptos para recibir canales extranjeros, a menos de, que dispongan del equipo necesario para decodificar la señal. (Fadul, 1985: 109110)

Mientras tanto, bien se podría argumentar que las recepciones directas mediante antenas parabólicas no constituyen, ni serán pronto un fenómeno masivo. Son muy costosas, difíciles de instalar e imponen la barrera del idioma. Por otro lado, las opciones temáticas que ofrecen no son fundamentalmente distintas de las proporcionadas por la programación de la televisión comercial actual. (Toussaint, 1984: 56) 
Empero, la entrada franca de mensajes e imágenes foráneas sin control alguno al territorio nacional, pone en jaque la soberanía sobre el espacio aéreo y las ondas electromagnéticas. (Toussaint, 1984: 56) En este sentido, se puede decir que la difusión directa "constituye una nueva amenaza para la penetración cultural utilizada por las centros industrializados mas desarrollados, ya que por medio de ellos se emiten mensajes y propaganda políticocultural y subliminal”, (García, 1984: 15) que distorsionan nuestra historia, cambian nuestra manera de ser, glorifican actitudes prepotentes de potencias hegemónicas y desvían el rumbo que nuestro país ha tomado en base a nuestra manera de entender la existencia del mundo y la vida”. (Osorio, 1984) De aquí, una vez mas la necesidad de estudiar los efectos profundos que ocasiona dicho fenómeno en nuestra sociedad y crear las acciones correctivas frente al mismo.

\section{B).- La dependencia tecnológica del exterior}

El panorama industrial de esta rama de las comunicaciones revela que si bien la mayor parte de las antenas en venta en el país son de fabricación nacional, no ocurre lo mismo con los equipos electrónicos de recepción de las señales y con la mayoría de los materiales para fabricar estaciones receptoras, salvo en el caso de empresa Resalt, S.A., que produce el 95 por ciento de los componentes de las antenas, y que resulta ser un caso de excepción. El resto de las empresas son, en su mayoría subsidiarias de las grandes casas fabricantes de estaciones receptoras en los EUA. Esto puede traer consigo un agudo déficit de divisas en este sector, no sólo por la importación de equipos y materiales, sino también por el pago de derechos de patente a las subsidiarias estadounidense, lo cual podría acrecentar este problema económico para México, especialmente, ahora que el ahorro de divisas es de vital importancia.

Asimismo, es pertinente apuntar que de todas las empresas que operan en el país, no se sabe cuáles son las que tienen permisos para la importación de equipos y cuáles introducen los aparatos por la frontera con los EVA violando todas las normas aduaneras y de control de la Secretaría de Comercio. (Fadul, 1985: 116-117)

Algunos expertos en la materia han advertido que la situación de la tecnología espacial en manos de consorcios multinacionales determina de antemano que, por lo menos, en la presente década, únicamente algunos de los países europeos, EUA, Japón y Canadá pongan en órbita sus propios satélites de difusión directa. De allí, se puede inferir que los países en desarrollo de las diversas regiones del planeta continuarán con su papel de 
consumidores de tecnología satelital, ya que es imposible pensar que tales naciones pueden llegar a ser en los próximos años, fabricantes de sofisticados sistemas satelitales, dadas otras prioridades de desarrollo dentro de sus economías. Se podría afirmar entonces que "el mercado para las compañías que dominan la industria espacial en el mundo occidental han comenzado a abrirse camino, sin ningún mecanismo legal que lo detenga. y seguramente tales consorcios tienen ya calculados sus ingresos y ganancias por tales acciones, al menos hasta finales del siglo XX”. (Fadul, 1985: 110-111)

Por esto mismo, nuestro país debe de considerar urgentemente la carencia de niveles adecuados en la investigación y desarrollo de tecnologías propias en materia de comunicaciones espaciales. Se sigue dependiendo de tecnologías importadas, que muchas veces no son las adecuadas en las condiciones geográficas o climatológicas de México. Los presupuestos asignados a estos rubros, no sólo por parte de las empresas fabricantes, sino del Estado mismo, han sido muy raquíticos, por lo que sería conveniente dedicar les mayor atención. De lo contrario, el país corre en este campo el riesgo de quedarse rezagado definitivamente al no obtener un lugar importante como productor de tecnología para telecomunicaciones. (Fadul, 1985: 111)

Estas solo son algunas de las repercusiones sociales puede traer consigo la difusión directa de señales vía satélite, y de ninguna manera constituyen un examen totalizador. Por ello, subrayamos la importancia central que tiene el seguir realizando dichos estudios en el país.

\section{Consideraciones finales}

La transmisión directa vía satélite es un fenómeno que en la actualidad se expande con gran celeridad y dinamismo por todo el territorio nacional, sin que exista una reglamentación competente que rija su operación en la sociedad mexicana. Lo único estipulado jurídicamente hasta el momento, es que el gobierno mexicano determina que las ondas hertzianas pertenecen a la Nación, como cualquiera de las vías de comunicación y que el uso de la atmósfera solo puede ser concesionado por la Secretaria de Comunicaciones y Transportes.

Estos principios son insuficientes para controlar el uso indiscriminado de estas vías de comunicación, ya que, por ejemplo, algunos mensajes transmitidos por radar son interceptados en las televisoras locales o en los aparatos caseros, lo que resta seguridad al Estado Mexicano al apropiarse de mensajes que no están destinados a todos los receptores comunes de información. (Montenegro, 1984: 7) 
Otro aspecto necesario de considerar es que los aparatos de antenas múltiples son de fabricación nacional, pero los llamados "rastreado res " son de importación. ¿Por qué la Dirección de Aduanas ha permitido esa compra incontrolada? y ¿ Por qué la Secretaría de Comercio y Fomento Industrial no ha dictado normas para su introducción? Al margen de estas consideraciones, creemos que ni siquiera por medio de un alto impuesto se podría resolver esta anomalía o controlar sus peligros. Lo más razonable sería que la Ley de Vías de Generales de Comunicación prevea esta modalidad de enajenación y la considere como una infraestructura de comunicaciones que de no ser supervisada por el Estado se puede convertir en un peligro potencial de consecuencias culturales impredecibles para la sociedad mexicana. (Montenegro, 1984: 7)

Por otro lado, dicha reglamentación se debe orientar al uso de las antenas parabólicas en términos de contenidos y no únicamente de instalaciones. (Toussaint, 1984: 56) Para ello, su funcionamiento deberá ser controlado estrictamente por la Secretaría de Gobernación, quien tiene la facultad de señalar medidas jurídicas que protejan a la cultura nacional. De esta forma, por ejemplo, la Secretaría de Gobernación puede solicitar apoyo a los técnicos mexicanos a fin de que la recepción de programas sea clasificada y solo se permitan aquellos que no perturben nuestra idiosincrasia, evitando la difusión de los que lesionan nuestras formas de vida o dañan sensiblemente la mente de la niñez y la juventud. (Osorio, 1984: 4)

Con la consideración de algunos de estos lineamientos jurídicos creemos que disminuiría en el país la anarquía en cuanto a la recepción de señales vía satélite por medio de las antenas parabólicas, y se ayudaría a contrarrestar los efectos nocivos de las transmisiones externas, fortaleciendo la identidad, cultura y soberanía nacionales.

\section{Bibliografía}

Alvares Lima, Antonio, 1984: Aprobaron los Diputados el Cobro por el Uso de Antenas Parabólicas, Excelsior 27 de Diciembre, México, D.F.: Excelsior

Barriga Chávez Ezequiel ,1982: En 1985 México Pondrá en Orbita su primer Satélite de Telecomunicaciones, entrevista con Clemente Pérez Correa, Director General de Telecomunicaciones en 1982, Rev. Hoy. México.

Constitución Politica de los Estados Unidos Mexicanos, Artículo 28, Tomado de Satélites de Comunicación en México, México D. F., 1985.

Diagnóstico de Telecomunicaciones Vía Satélite ,1981: en: Diagnóstico de la Comunicación Social en México, Tomo IX, Parte B, México, D.F: 
Coordinación General de Comunicación Social de la Presidencia de la República.

Diario Oficial de la Federación ,1985: 21 de Enero de 1985, México, D.F.: Secretaría de Gobernación

Especificaciones para la Recepción de Señales Via Satélite de la Empresa Diseños Electromecánicos, S.A. ,1985: Documento Especial, Diseños Electromecánicos, S.A., México, D.F.

Especificaciones para la Recepción de Señales Via Satélite de la Empresa Telesat, S.A. de C. V. ,1985: Documento Especial, TELESAT, S.A. de C.V., México, D.F.

Fadul, Lígia María ,s/f: Los Satélites de Comunicación y el Caso de México, en Arriaga, Patricia, La Revolución Informática en México, , México, D.F.: CEESTEM Nueva Imagen.

Fadul, Lígia María; Fernández Christlieb, Fátima; Schmucler, Héctor ,1985: Satélites de Comunicación en México, Revista Comunicación y Cultura No. 13, Marzo, México, D.F.: Universidad Autónoma Metropolitana, Unidad Xochimilco

Fernández Christlieb, Fátima ,1985: Canal 7 ¿Para Qué? "' La Jornada, México, D.F., 2 de marzo.

Fernández Christlieb, Fátima ,1984: Génesis del Sistema Morelos, Perfil de La Jornada, México, D.F., 12 de diciembre.

García Moreno, Víctor, 1984: Constituyen las Antenas Parabólicas un Peligro para la Cultura Nacional: UNAM; El Universal, México, D.F., 2 de diciembre.

Gil, Antonio, 1984: Dictamen de la Cámara de Senadores: Exclusivo del Estado Usar Satélites de Comunicación, El Sol de México, México, D.F., 15 de diciembre.

Kanahuati Gómez, Jorge, 1982: Radiodifusión Directa por Satélite, Subdirección General de Coordinación Regional, Documento Interno, PRI, México, D.F., 29 de Marzo.

Los Satélites de Comunicación y el Caso de México.

Montenegro, Manuel Roberto, 1984: Antenas Parabólicas, Penetración Peligrosa, Excelsior, 14 de julio.

Osorio Marbán, Miguel, 1984: Antenas Parabólicas y Gobernación, El Sol de México, D.F., 11 de octubre.

Osorio Marbán, Miguel, 1984: Antenas Parabólicas y Gobierno. El Sol de México, 11 de octubre.

Propaganda de la Empresa Sekore 2000, S.A: Documento de Distribución, 1985: Sekore 2000, México, D.F. 
Rodríguez Galán, Azucena Patricia ,1984: Los Satélites Internacionales de Comunicación, Documento Interno, Departamento de Comunicación, Universidad Iberoamericana, México, D.F.

Schmucler, Héctor 25 años de Satélites Artificiales, Revista Comunicación y Cultura, No. 9, Universidad Autónoma Metropolitana-Xochimilco, México, D.F.

Toussaint, Florance ,1984: Antenas Parabólicas y Soberanía, Revista Proceso No. 254, México, D.F., 24 de diciembre.

Toussaint, Florance ,1984: Antenas Parabólicas y Soberanía, Revista Proceso, 24 de diciembre.

Un Sistema de Radiodifusión Directa Aplicable al Continente Americano, 1981: Vicepresidencia de Expansión de Televisa, Primer Seminario de Comunicaciones y Radiodifusión Directa por Satélite, Documento Interno, México, D.F., Agosto. 